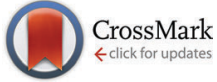

Cite this: Phys. Chem. Chem. Phys. 2015, 17, 27488

Received 12th August 2015 Accepted 16th September 2015

DOI: $10.1039 / c 5 c p 04803 f$

www.rsc.org/pccp

\title{
Dynamics of ultrathin gold layers on vitreous silica probed by density functional theory
}

\author{
Carolin Hühn, Lothar Wondraczek and Marek Sierka*
}

\begin{abstract}
The structure and properties of Au ultrathin films on hydroxyl-free and hydroxylated silica glass surfaces are investigated using ab initio molecular dynamics simulations. Substantial surface structure dependence of $\mathrm{Au}$ agglomeration behavior (solid-state dewetting) is found. On hydroxyl-free surfaces, the Au film virtually undergoes instantaneous agglomeration accompanied by the formation of voids exposing a bare silica glass surface. In contrast, simulated annealing of the Au film on hydroxylated surface models leaves its structure unchanged within the simulation time. This points to a key role of reactive defect sites in the kinetics of solid-state dewetting processes of metals deposited on the glass surface. Such sites are important for initial void nucleation and formation of metal clusters. In addition, our calculations demonstrate the crucial role of the appropriate inclusion of dispersion interactions in density functional theory simulations of metals deposited on glass surfaces. For defective, hydroxyl-free glass surfaces the dispersion correction accounts for $35 \%$ of the total adhesion energy. The effect is even more dramatic for hydroxylated glass surfaces, where adhesion energies are almost entirely due to dispersion interactions. The Au adhesion energies of 200 and $160 \mathrm{~kJ}(\mathrm{~mol} \mathrm{~nm})^{2}$ calculated for hydroxylated glass surfaces are in good agreement with the experimental data.
\end{abstract}

\section{Introduction}

Ultrathin metal layers and metal clusters deposited on silica and silicate glasses play an important role in a broad variety of technical applications ranging from architecture and automotive glazing to electronic devices, sensor technology and catalysis. ${ }^{1-8}$ In particular, continuous layers, particulate deposits and clusters of noble metals have attained key importance due to their high imaginary part of the refractive index and, consequently, high optical reflectivity along with the specific position of their plasmonic resonance frequency. This enables a precise control of spectral reflection and transmission (e.g., for low-emissivity and solar protection windows) as well as other properties such as their catalytic or antimicrobial activity. ${ }^{9,10}$ Tailoring of the metal layer structure and thickness (usually between a monolayer and a few $\mathrm{nm}$ ), controlling the formation of atomic agglomerates, cluster deposits or nanoparticles, is the essential step in all these applications. It has been shown that film homogeneity and noble metal agglomeration behavior very strongly depend on subtle variations in the substrate conditions such as the degree of surface hydroxylation. ${ }^{11}$

During the deposition process but also during off-line annealing or long-term operation thin metal films can disintegrate

Otto Schott Institute of Materials Research, Friedrich Schiller University of Jena, Löbdergraben 32,07743 Jena, Germany.E-mail: marek.sierka@uni-jena.de into particles, even upon annealing at temperatures well below the melting point, for example, through solid-state dewetting. ${ }^{12-18}$ It is assumed that this process proceeds in three steps: (i) void initiation, (ii) void growth and (iii) void coalescence and particle formation. ${ }^{17}$ It has further been postulated that the initial voids form at grain boundaries, vacancies, impurities or pores. ${ }^{15-17}$ However, the role of surface defect sites of substrates in the solid-state dewetting process has been largely disregarded in this context. The present hypothesis is that voids, initiated by any nucleation center, grow towards the film surface. The time needed for this process is called incubation time, ranging typically from some minutes to hours, depending on film thickness and annealing temperature. ${ }^{17}$ After breaking through the entire film, the voids grow and coalesce to form metal particles. However, Seguini $e t a l .^{18}$ have found that ultrathin Au films ( 0.5 to $6 \mathrm{~nm}$ thickness) deposited at room temperature on amorphous $\mathrm{SiO}_{2}$ by means of e-beam evaporation spontaneously aggregate, forming nanoparticles without any additional thermal treatment.

Computational studies of noble metal agglomeration on silica surfaces are so far scarce. ${ }^{19}$ To our best knowledge $a b$ initio investigations of $\mathrm{Au}$ on $\mathrm{SiO}_{2}$ surfaces were limited to a single $\mathrm{Au}$ atom or an Au dimer interacting with various cluster or crystalline models of silica. ${ }^{20-23}$ In the present study the structure and properties of Au ultrathin films on hydroxyl-free and hydroxylated silica glass surfaces are investigated for the first time using $a b$ initio molecular dynamics (MD) simulations. 


\section{Computational details}

\subsection{General simulation procedure}

Fig. 1 depicts the simulation procedure applied in this work. As the first step models of bulk silica glass were generated through simulated melting and quenching at the ReaxFF force field ${ }^{24,25}$ level, starting from $\beta$-cristobalite supercells. The reliability of the models was assessed by comparison of the calculated structure and properties with the experimental data for silica glass. In the second step, slab models of hydroxyl-free glass surfaces were cut out of the bulk glass models, annealed using the ReaxFF force field and subsequently optimized at the density functional theory (DFT) level. From this, hydroxylated surface models were obtained by saturating all surface defects with oxygen and hydrogen atoms. Finally, a gold monolayer was deposited on each such surface model and MD simulations at the DFT level were carried out. The final equilibrated structures were optimized at the DFT level and used to evaluate gold-glass adhesion energies.

\subsection{Methods}

Structural optimizations and MD simulations at the ReaxFF force field ${ }^{24}$ level were carried out using parameters reported in ref. 25. For all ReaxFF MD simulations, an NVT ensemble with a 0.5 fs time step and linear velocity scaling for cooling procedures was used, employing the LAMMPS (Large-scale Atomic/Molecular Massively Parallel Simulator) program. ${ }^{26}$

Structural optimizations and MD simulations at the DFT level were performed using the Perdew-Burke-Ernzerhof (PBE) exchange-correlation functional ${ }^{27}$ and the projector-augmented plane-wave (PAW) ${ }^{28,29}$ method as implemented in the VASP (Vienna $a b$ initio Simulation Package) program. ${ }^{30-33}$ Unless stated otherwise, a $250 \mathrm{eV}$ cutoff for the plane wave basis set and the $\Gamma$ point for the integration ${ }^{34}$ of the first Brillouin zone were used. All MD simulations at the DFT level were performed with a 1 fs time step and linear velocity scaling applied for annealing procedures. For surface models the Grimme dispersion correction (DFT-D3) ${ }^{35}$ was added. It has been shown that the DFT-D3 method achieves accuracy comparable with $\operatorname{CCSD}(\mathrm{T})$. For Au clusters DFT-D3 yields relative energies that are within $8 \mathrm{~kJ} \mathrm{~mol}^{-1}$ of the $\operatorname{CCSD}(\mathrm{T})$ reference values. $^{35}$

\subsection{Bulk glass models}

As noted above, bulk glass models were prepared by a simulated melting and quenching process at the ReaxFF force field level. First, supercells of $\beta$-cristobalite were constructed as summarized in Table 1. Using MD simulations, each supercell was equilibrated at $8000 \mathrm{~K}$ for 175 ps. At every 50 ps, 75 ps, 100 ps, 125 ps, 150 ps, and 175 ps a structure snapshot was taken. The snapshots were cooled down to $300 \mathrm{~K}$ within $2 \mathrm{~ns}$, and optimized. The most stable structure for each supercell size, denoted as G2-G8 ( $c f$. Table 1), was used for further calculations. G2 and G3 were subsequently optimized at the DFT level.

Harmonic vibrational frequencies for G2 were calculated using the density functional perturbation theory ${ }^{36}$ as implemented in VASP. Infrared intensities were obtained from the matrix of Born effective charges employing the formula given by Baroni et al. ${ }^{37}$ The vibrational frequencies were scaled ${ }^{38}$ by 0.9825 . This scaling factor was determined by fitting calculated infrared (IR) frequencies to the experimental data. ${ }^{39}$

The stiffness tensor $\mathbf{C}$ for $\mathbf{G} 2$ was calculated at the DFT level using an energy cutoff of $400 \mathrm{eV}$ for the plane wave basis set. Young's modulus $E$, shear modulus $G$ and the Poisson ratio $\nu$ were determined under the conditions of an isotropic material, which leads to the following expression for $\mathbf{C}$ :

$$
\mathbf{C}=\left[\begin{array}{llllll}
C_{1} & C_{2} & C_{2} & 0 & 0 & 0 \\
C_{2} & C_{1} & C_{2} & 0 & 0 & 0 \\
C_{2} & C_{2} & C_{1} & 0 & 0 & 0 \\
0 & 0 & 0 & C_{3} & 0 & 0 \\
0 & 0 & 0 & 0 & C_{3} & 0 \\
0 & 0 & 0 & 0 & 0 & C_{3}
\end{array}\right],
$$

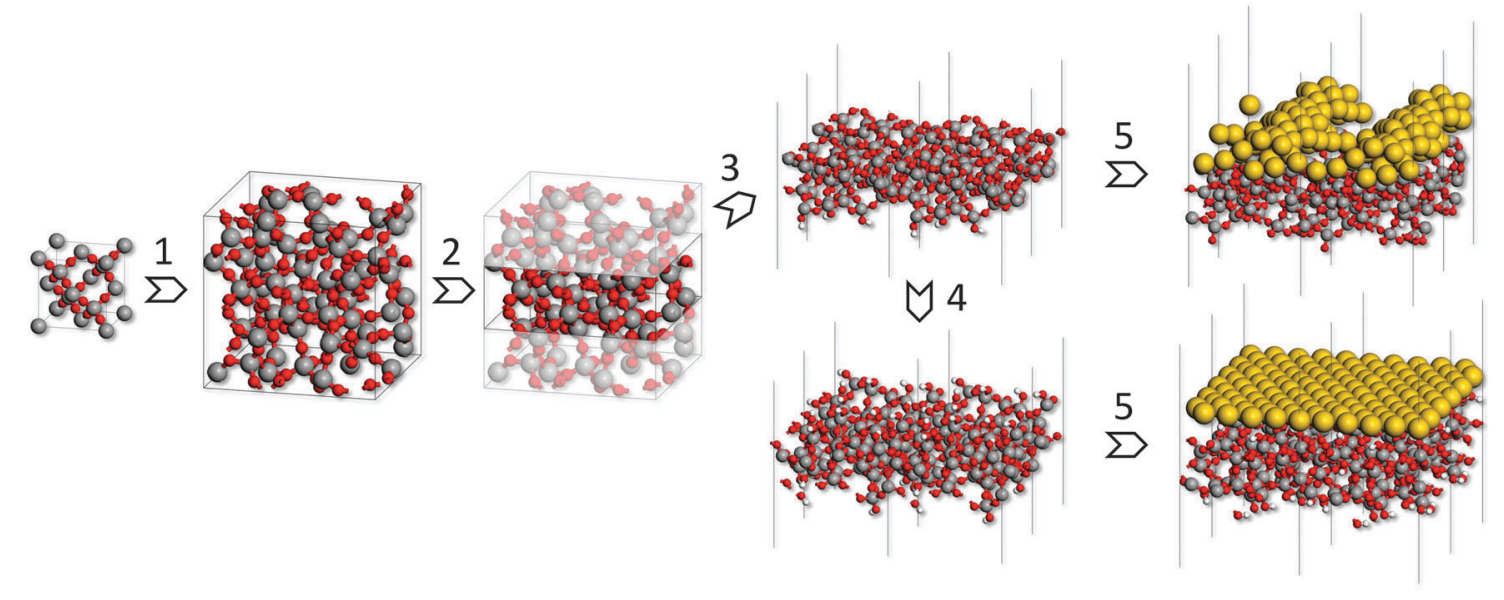

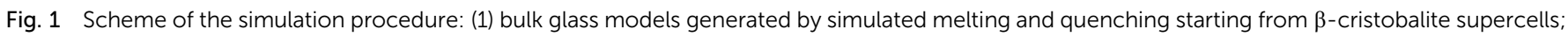

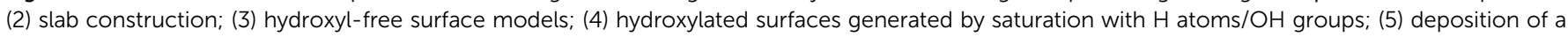
gold monolayer and MD simulations at the DFT level. (Si: grey, O: red, H: white, Au: yellow). 
Table 1 Bulk glass models: initial $n \times n \times n \beta$-cristobalite supercells, cell dimension $l(\mathrm{~nm})$ and the number of $\mathrm{SiO}_{2}$ units $N$ per supercell

\begin{tabular}{lllr}
\hline Model & Supercell & $l$ & \multicolumn{1}{c}{$N$} \\
\hline G2 & $2 \times 2 \times 2$ & 1.42 & 64 \\
G3 & $3 \times 3 \times 3$ & 2.14 & 216 \\
G4 & $4 \times 4 \times 4$ & 2.85 & 512 \\
G5 & $5 \times 5 \times 5$ & 3.56 & 1000 \\
G6 & $6 \times 6 \times 6$ & 4.27 & 1728 \\
G7 & $7 \times 7 \times 7$ & 4.98 & 2744 \\
G8 & $8 \times 8 \times 8$ & 5.70 & 4096
\end{tabular}

with

$$
\begin{aligned}
& C_{1}=\frac{E(1-\nu)}{(1+\nu)(1-2 \nu)}, \quad C_{2}=\frac{E \nu}{(1+\nu)(1-2 \nu)}, \\
& C_{3}=G=\frac{C_{1}-C_{2}}{2} .
\end{aligned}
$$

The values of $E$ and $\nu$ were then expressed accordingly as

$$
E=\frac{\left(C_{1}-C_{2}\right)\left(C_{1}+2 C_{2}\right)}{C_{1}+C_{2}}, \quad \nu=\frac{C_{2}}{C_{1}+C_{2}} .
$$

\subsection{Pristine glass surface models}

Two slab models of pristine glass surfaces (denoted as S1 and S2) were constructed by cutting two-dimensional, about $9 \AA$ A thick slabs out of the $\mathbf{G} 2$ model. These slabs were optimized, annealed at $1500 \mathrm{~K}$, cooled down to $300 \mathrm{~K}$ within $2 \mathrm{~ns}$ and subsequently optimized using the ReaxFF force field. Three-dimensional slab models for DFT calculations were prepared by adding a vacuum gap of $15 \AA$ followed by structural optimization at the DFT level.

\subsection{Hydroxylated glass surface models}

Hydroxylated surface models were prepared by saturating all surface defects (threefold coordinated silicon $\left(\mathrm{Si}^{3}\right)$, non-bridging oxygen (NBO) and three-membered Si-O rings (3-ring)) of $\mathbf{S 1}$ and S2 with oxygen and hydrogen atoms. These hydroxylated slab models hS1 and hS2 were optimized, annealed at $300 \mathrm{~K}$ within $2 \mathrm{~ns}$ and subsequently optimized using the ReaxFF force field. A vacuum gap of $15 \AA$ was added and the models were optimized at the DFT level.

\subsection{Gold monolayer models}

An fcc gold unit cell was optimized at the DFT level using a $10 \times$ $10 \times 10$ Monkhorst-Pack grid for the integrations of the first Brillouin zone and a $400 \mathrm{eV}$ cutoff for the plane wave basis set. To deposit an Au film a single (111) $14.5 \times 15 \AA$ Au monolayer containing 30 atoms was cut out of the bulk structure. It was compressed to $14.2 \times 14.2 \AA$ to fit the dimension of the glass surface models and placed at a distance of approximately $3.5 \AA$ over the surface. The structures were subsequently optimized at the DFT level keeping the cell parameters constant. The glass surface atoms within the upper $5 \AA$ were allowed to relax with the remaining atoms fixed to their original positions so as to mimic bulk glass behavior. Next, the systems were annealed from $1400 \mathrm{~K}$ to $300 \mathrm{~K}$ within 5 ps using MD calculations at the DFT level and were subsequently optimized.
The adhesion energy, $E_{\text {ad }}$, of the gold film was calculated as

$$
E_{\mathrm{ad}}=\frac{E_{\mathrm{Au}}+E_{\mathrm{SiO}_{2}}-E_{\mathrm{Au} / \mathrm{SiO}_{2}}}{x}
$$

where $E_{\mathrm{Au} / \mathrm{SiO}_{2}}$ is the total energy of the annealed and optimized gold/glass interface model. $E_{\mathrm{Au}}$ and $E_{\mathrm{SiO}_{2}}$ refer to the total energies of the gold layer and of the clean substrate, respectively, and $x$ is the number of gold atoms per supercell (for $E_{\text {ad }}$ in kJ $(\mathrm{mol} \mathrm{Au})^{-1}$ ) or the surface area (for $E_{\mathrm{ad}}$ in $\left.\mathrm{kJ}\left(\mathrm{mol} \mathrm{nm}^{2}\right)^{-1}\right)$. With this definition, adhesion is energetically favorable if the value of $E_{\text {ad }}$ is positive. The dispersion energy contribution $E_{\mathrm{dis}}$ of the interface was calculated applying eqn (4) and substituting Grimme correction contributions for the total energies.

\section{Results and discussion}

\subsection{Glass models}

Simulations of amorphous materials usually employ semiamorphous periodic models. Within this approximation a proper description of the amorphous nature requires sufficiently large simulation cells. Therefore, a number of differently sized cells were generated to identify the limit of percolation at which the ensemble size is sufficiently large to describe the properties of bulk silica glass. Fig. 2 shows two examples of the glass models summarized in Table 1. The differences between the energy per $\mathrm{SiO}_{2}$ unit of models with different sizes are very small, within 1-4 kJ $\left(\mathrm{mol} \mathrm{SiO}_{2}\right)^{-1}$, for both ReaxFF and DFT methods. This indicates that already the smallest model is suitable to describe vitreous silica, and also validates the accuracy of the ReaxFF force field. For further assessment of the reliability of our model, selected properties were calculated and compared to the experimental data. Fig. 3 shows the comparison of the total correlation function of $\mathbf{G 2}$ with the experimental neutron diffraction data for silica glass. ${ }^{40}$ The agreement between calculated and experimental peak positions is very good. The first peak at $1.61 \AA$ corresponds to the average distance between the nearest $\mathrm{Si}$ and $\mathrm{O}$ atoms, the second peak at $2.64 \AA$ to the average distance between the nearest O atoms and the peak at $3.08 \AA$ to the average distance between the nearest $\mathrm{Si}$ atoms. Table 2 summarizes the average interatomic distances and average bond angles for both ReaxFF and DFT optimized models. Both methods reproduce the experimental average distances very well, with DFT slightly overestimating and ReaxFF slightly underestimating the distances. The absolute (a)

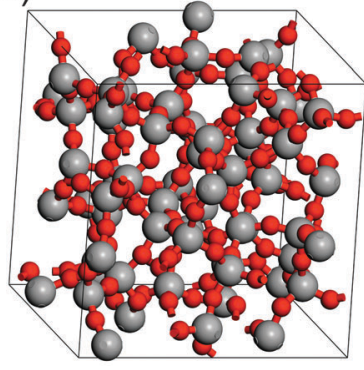

(b)

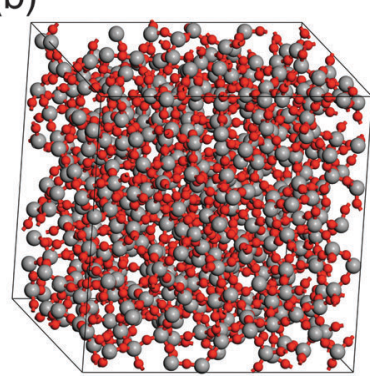

Fig. 2 Bulk silica glass models G2 (a) and G5 (b) (see Table 1; Si: grey, O: red). 


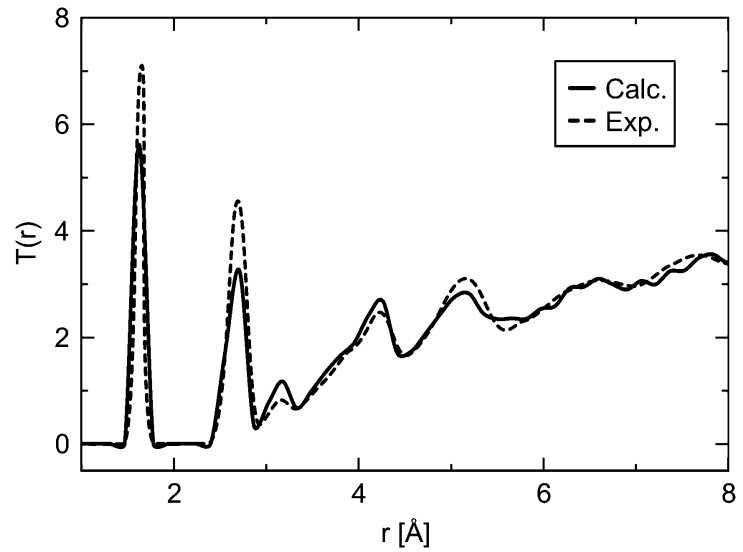

Fig. 3 Comparison of the total correlation function $T(r)$ of G2 (solid line, see Table 1) with the experimental ${ }^{40}$ data (dashed line).

Table 2 Calculated (DFT for G2 and G3, ReaxFF for G2-G8) and experimental average bond distances $d(\AA)$ and average bond angles (deg)

\begin{tabular}{lccl}
\hline Parameter & DFT & ReaxFF & Exp. \\
\hline$d_{\text {Si-O }}$ & 1.63 & 1.58 & $1.61^{a}$ \\
$d_{\mathrm{O}-\mathrm{O}}$ & 2.66 & 2.55 & $2.64^{a}$ \\
$d_{\text {Si-Si }}$ & 3.12 & 3.09 & $3.08^{a}$ \\
O-Si-O & 109.4 & 109.3 & $109.33^{b}$ \\
$\mathrm{Si}-\mathrm{O}-\mathrm{Si}$ & 151.7 & 155.6 & $151.5-152.2^{c}$ \\
${ }^{a}$ Ref. $40 .{ }^{b}$ Ref. $42 .{ }^{c}$ Ref. 41. & &
\end{tabular}

errors of the average interatomic distances are between 0.02 and $0.09 \AA$ And the relative errors are between 1.3 and $3.4 \%$. The average bond angles are in very good agreement with the experimental values. $^{41,42}$

Fig. 4 shows the calculated and experimental ${ }^{39}$ IR transverseoptic spectra of silica glass with the Si-O-Si rocking vibration at about $457 \mathrm{~cm}^{-1}$, the Si-O-Si symmetrical stretching vibration at about $810 \mathrm{~cm}^{-1}$ and the $\mathrm{Si}-\mathrm{O}-\mathrm{Si}$ asymmetrical stretching

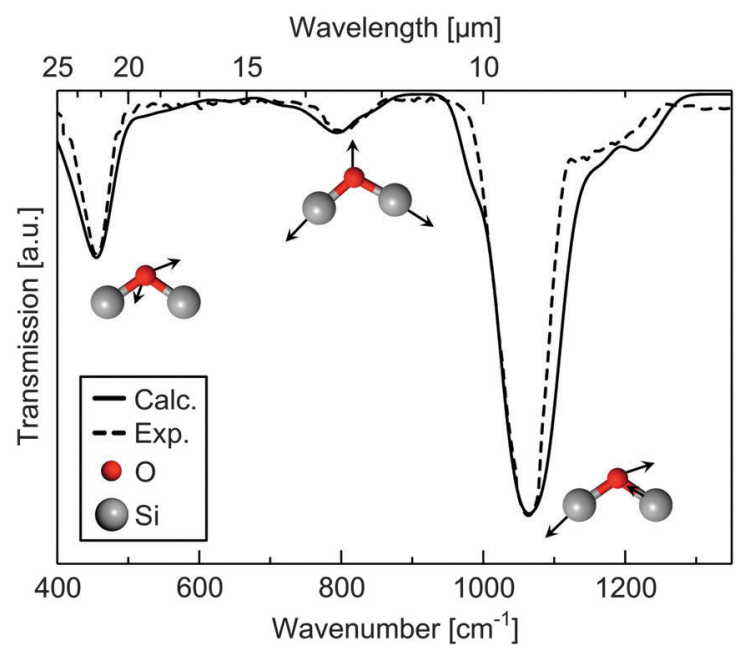

Fig. 4 Experimental ${ }^{39}$ (dashed line) and calculated (G2 model, solid line) infrared spectrum of bulk silica glass. The calculated spectrum is convoluted using a Gaussian line shape function with a half-width of $12 \mathrm{~cm}^{-1}$. vibration at approximately $1076 \mathrm{~cm}^{-1}$. The calculated values are in very good agreement with the experimental ${ }^{39}$ data. The positions of the adsorption bands are reproduced within $2-6 \mathrm{~cm}^{-1}$.

The calculated Young's modulus, the shear modulus and the Poisson ratio of G2 are $95 \pm 9 \mathrm{GPa}, 35 \pm 1 \mathrm{GPa}$ and $0.176 \pm 0.02$, respectively. The calculated values agree well with the experimental data of $73.5 \mathrm{GPa}, 31.5 \mathrm{GPa}$ and $0.17,{ }^{43}$ respectively.

In summary, the properties calculated even for the smallest G2 model containing $64 \mathrm{SiO}_{2}$ units are in very good agreement with the experimental data available for bulk silica glass. This confirms the validity of our procedure for generating glass models.

\subsection{Glass surfaces}

Surface properties of real glasses vary widely depending on the environmental conditions. In a very moist environment, silicate glass surfaces are typically fully hydroxylated, whereas heattreated $\left(\sim 1200{ }^{\circ} \mathrm{C}\right)$ glass in a vacuum chamber displays a hydroxyl-free surface. ${ }^{44}$ Therefore, two limiting cases of silica glass surfaces were considered here: fully hydroxylated and hydroxyl-free surfaces. The corresponding surface models are shown in Fig. 5. Due to the limited size of the models they do not include low concentration defects, such as two membered $\mathrm{Si}-\mathrm{O}$ rings (concentration $0.2-0.4 \mathrm{~nm}^{-2}$ ). ${ }^{45}$

Table 3 compares the calculated and experimental properties of hydroxylated surfaces. The concentration of surface silanol groups for both hS1 and hS2 models of 4.4 and $4.9 \mathrm{~nm}^{-2}$, respectively, is in very good agreement with the experimental value $^{44}$ of $4.9 \pm 0.05 \mathrm{~nm}^{-2}$. The slight deviation of $0.5 \mathrm{~nm}^{-2}$ for hS1 is due to the relatively small surface area of the models, hence, the removal of one silanol group results in a concentration error of $0.5 \mathrm{~nm}^{-2}$. However, the ratio of geminal silanols to the total number of silanol groups of 0.22 and 0.1 for both hS1 and $\mathbf{h S 2}$, respectively, is in very good agreement with the range of $0.08-0.24$, which was observed in experiments. ${ }^{46,47}$

Table 4 shows calculated concentrations of $\mathrm{NBO}$ and $\mathrm{Si}^{3}$ surface sites as well as 3-membered $\mathrm{Si}-\mathrm{O}$ rings for hydroxyl-free glass surface models S1 and S2. NBOs are only present in S1, whereas $\mathrm{Si}^{3}$ and 3-membered $\mathrm{Si}-\mathrm{O}$ rings are included in both models. There are only few qualitative $e^{45,48,49}$ and quantitative ${ }^{50-52}$ experimental data concerning the defect concentration and structure of hydroxyl-free silica surfaces. Bobyshev and Radzigo,51 determined the concentration of $\mathrm{Si}^{3}$ and NBO centers on activated silica surfaces as 0.02 and 0.02 to $0.04 \mathrm{~nm}^{-2}$, respectively. McCrate et al. ${ }^{52}$ found a slightly higher value of approximately $10^{-1} \mathrm{~nm}^{-2}$ for $\mathrm{Si}^{3}$ centers. The limited size of our surface models prevents achieving such low defect concentrations. Already one defect site per surface unit cell results in a concentration of $0.49 \mathrm{~nm}^{-2}$ and modeling even lower ones would require prohibitively large simulation cells.

\subsection{Gold monolayer on glass surfaces}

Simulated annealing of gold monolayers on hydroxyl-free surface models S1 and $\mathbf{S} 2$ leads to its virtually instantaneous agglomeration and formation of voids exposing a bare glass surface as demonstrated for the final structures in Fig. $6 a$ and b. The analysis of MD 
(a)
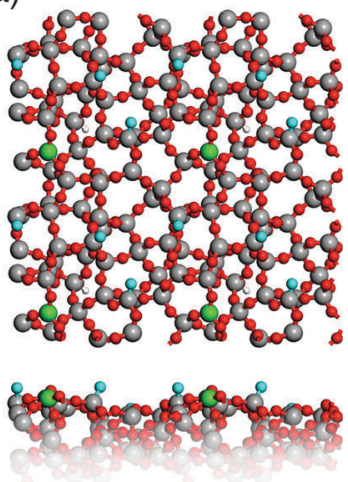

(b)

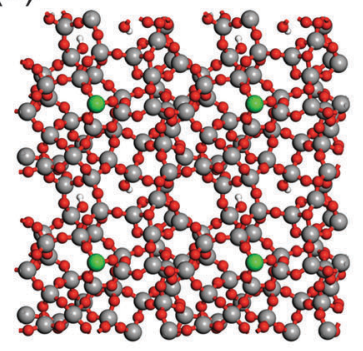

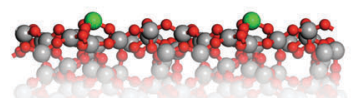

(c)

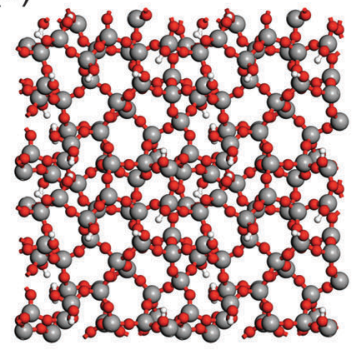

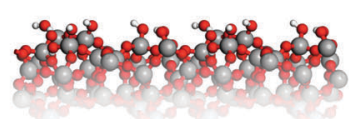

(d)
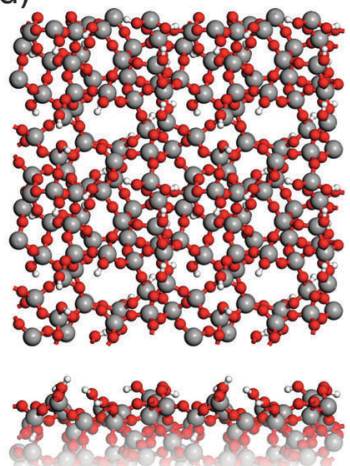

Fig. 5 Top and side view of glass surface models. (a) and (b) Hydroxyl-free $\mathbf{S 1}$ and S2; (c) and (d) hydroxylated hS1 and hS2 (Si: grey, O: red, H: white, Si³. green, NBOs: blue).

Table 3 Calculated (hS1 and hS2 surface models) and experimental concentration of silanol groups $\left(\mathrm{C}_{\mathrm{SiOH}}, \mathrm{nm}^{-2}\right)$ along with the ratio of geminal silanols to the total number of silanols $\left(\mathrm{Si}(\mathrm{OH})_{2}: \mathrm{SiOH}_{\text {tot }}\right)$ for hydroxylated glass surfaces

\begin{tabular}{llll}
\hline & hS1 & hS2 & Exp. \\
\hline$\left.c_{\mathrm{SiOH}}\right)_{2}: \mathrm{SiOH}_{\text {tot }}$ & 4.4 & 4.9 & $4.9 \pm 0.05^{a}$ \\
$\mathrm{Si}(\mathrm{OH})_{2}$ & 0.22 & 0.1 & $0.08-0.24^{b, c}$ \\
${ }^{a}$ Ref. $44 .{ }^{b}$ Ref. $46 .{ }^{c}$ Ref. 47. & & \\
\hline
\end{tabular}

Table 4 Calculated concentrations of non-bridging oxygen $\left(\mathrm{NBO}, \mathrm{nm}^{-2}\right.$ ), three-coordinated silicon $\left(\mathrm{Si}^{3}, \mathrm{~nm}^{-2}\right)$ and three-membered $\mathrm{Si}-\mathrm{O}$ ring (3-ring, $\mathrm{nm}^{-2}$ ) surface defects in hydroxyl-free glass surface models $\mathbf{S 1}$ and $\mathbf{S 2}$

\begin{tabular}{lll}
\hline & S1 & S2 \\
\hline $\mathrm{NBO}$ & 1.48 & 0 \\
$\mathrm{Si}^{3}$ & 0.49 & 0.49 \\
3-ring & 0.99 & 2.47
\end{tabular}

trajectories with snapshots shown in Fig. 7 reveals that voids are initiated by opening the Au layer at the NBO sites with the
$\mathrm{Si}^{3}$ centers located beneath the forming Au clusters. The growing voids gradually expose defect-free parts of the silica surface with the NBO sites remaining in close contact with the edge of the voids and $\mathrm{Si}^{3}$ remaining beneath $\mathrm{Au}$ clusters. In the final, optimized structures (Fig. 6a and b) the corresponding bond distances are in average 2.17 $\AA$ and $2.33 \AA$ for Au-NBO and $\mathrm{Au}-\mathrm{Si}^{3}$, respectively. These findings indicate that defect sites such as $\mathrm{NBO}$ and $\mathrm{Si}^{3}$ centers on hydroxyl-free glass surfaces play a key role in the solid-state dewetting processes by providing centers for initial void nucleation and cluster formation. In addition, the strong interaction with the defect sites stabilizes the forming Au clusters.

Our results are supported by experimental ${ }^{18}$ and theoretical ${ }^{19}$ data. Seguini et al. ${ }^{18}$ found that ultrathin $\mathrm{Au}$ films $(0.5$ to $6 \mathrm{~nm}$ thickness) deposited at room temperature on amorphous $\mathrm{SiO}_{2}$ by means of e-beam evaporation spontaneously aggregate, forming nanoparticles without any additional thermal treatment. In addition, rapid $\mathrm{Au}$ agglomeration on hydroxyl-free (0001) $\alpha$-quartz surfaces has also been found by Kuo et al. ${ }^{19}$ using MD simulations employing interatomic potential functions. (a)

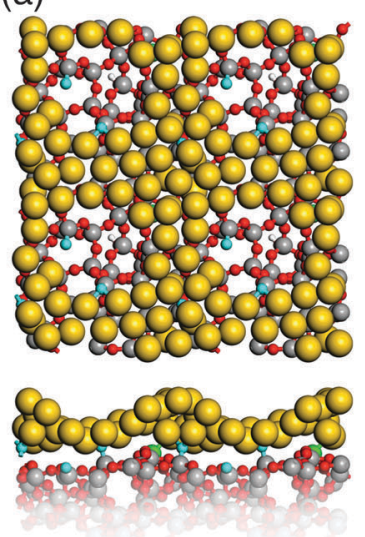

(b)
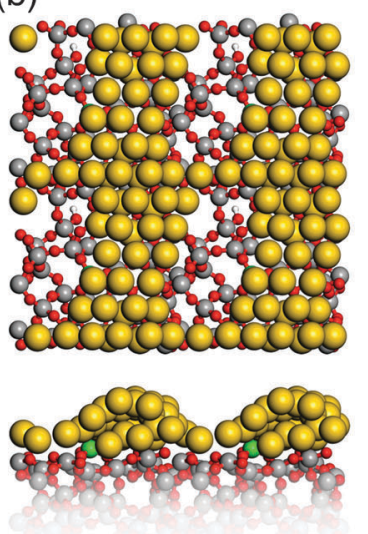

(c)
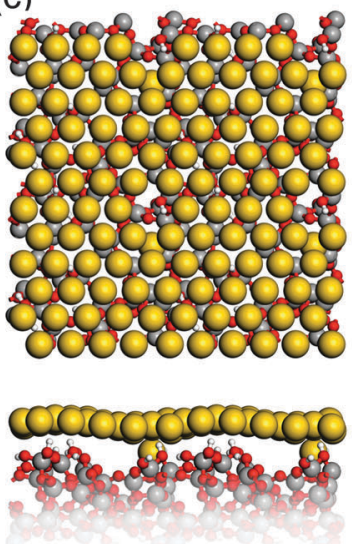

(d)
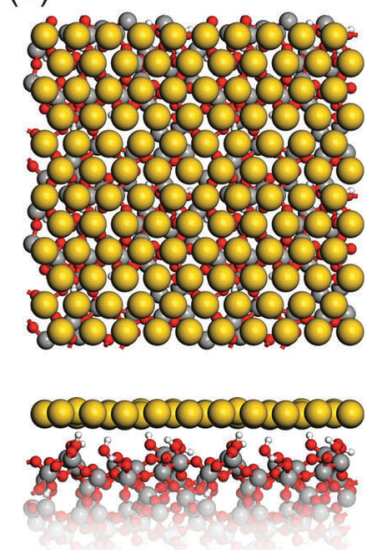

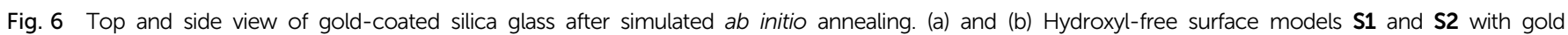

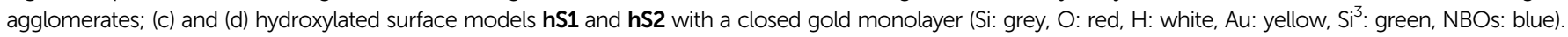


(a)
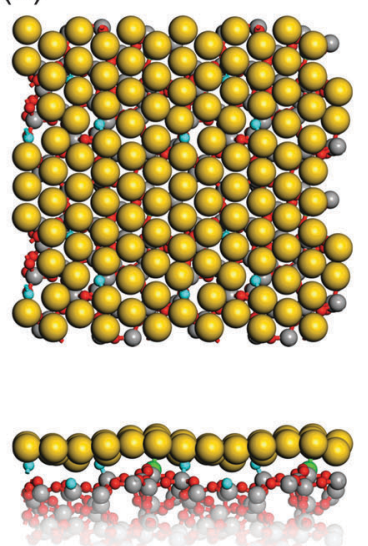

(b)
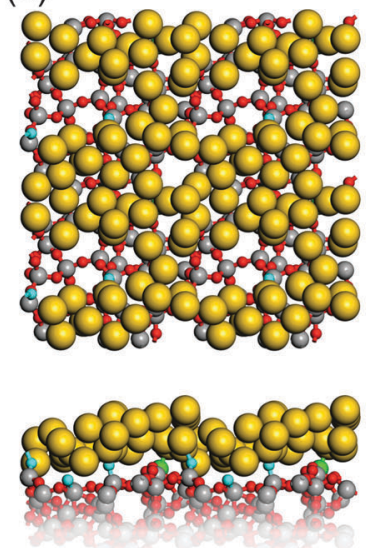

(c)
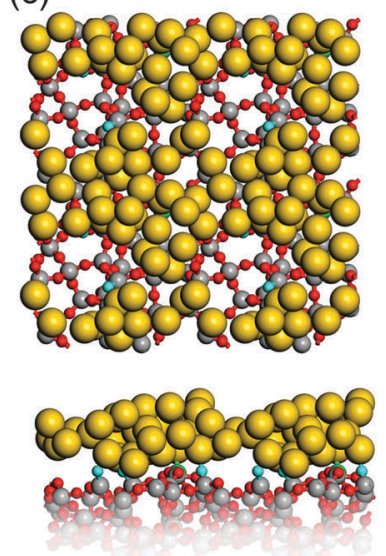

(d)
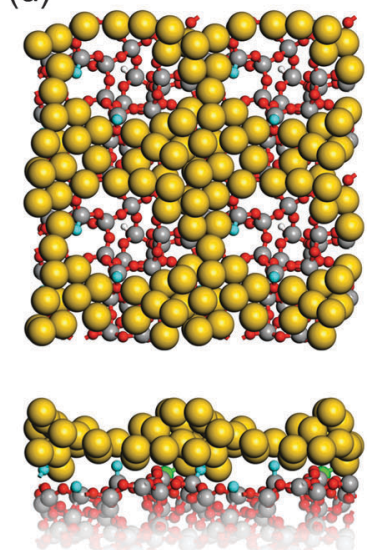

Fig. 7 Snapshots of the MD trajectory for simulated annealing of an Au monolayer on the S1 glass surface model: (a) $1400 \mathrm{~K}$, (b) $1350 \mathrm{~K}$, (c) $1300 \mathrm{~K}$, and (d) 900 K (Si: grey, O: red, Au: yellow, $\mathrm{Si}^{3}$ : green, NBOs: blue).

In contrast to hydroxyl-free surfaces, simulated annealing of a gold monolayer on hydroxylated surface models hS1 and hS2 leaves its structure virtually unchanged within the simulation time (see Fig. 6c and d). This finding is consistent with the observed prolonged incubation times for agglomeration of thin $\mathrm{Au}$ layers on hydroxylated silica glass surfaces. ${ }^{14,17}$ In light of our results, it appears that this behavior can be attributed to the absence of nucleation centers in the form of surface defects, as they are saturated on hydroxylated surfaces.

Table 5 shows calculated adhesion energies of the final $\mathrm{Au}$ layers on the hydroxyl-free and hydroxylated glass surface models along with the corresponding dispersion contributions. For hydroxyl-free models $\mathbf{S} 1$ and $\mathbf{S} 2$ the calculated adhesion energies are 40.2 and $33.3 \mathrm{~kJ}(\mathrm{~mol} \mathrm{Au})^{-1}$, respectively. The dispersion energy contribution to the adhesion energies of $35 \%$ on average is quite significant. In the case of hydroxylated models hS1 and hS2 the adhesion energies of 13.5 and $10.8 \mathrm{~kJ}(\mathrm{~mol} \mathrm{Au})^{-1}$, respectively, are substantially lower and consist almost entirely of the dispersion contribution. The calculated $\mathrm{Au}$ adhesion energies per area, 200 and $\left.160 \mathrm{~kJ}(\mathrm{~mol} \mathrm{~nm})^{2}\right)^{-1}$ for hS1 and hS2, respectively, are in a good agreement with the experimental value of $\left.216 \mathrm{~kJ}(\mathrm{~mol} \mathrm{~nm})^{2}\right)^{-1}$ measured for gold films on hydroxylated silica glass surfaces. ${ }^{15}$

To our best knowledge, the present study represents the first investigation of the behavior of Au layers on realistic models of vitreous silica surfaces using $a b$ initio methods. Previous theoretical investigations were limited to one $\mathrm{Au}$ atom or an $\mathrm{Au}$ dimer interacting with various models of the silica surface.

Table 5 Calculated adhesion energies $E_{\text {ad }}$ and their dispersion contributions $E_{\text {dis }}\left(\mathrm{kJ}(\mathrm{mol} \mathrm{Au})^{-1}\right.$ and $\mathrm{kJ}(\mathrm{mol} \mathrm{nm})^{-1}$ in brackets) of gold on hydroxylated (hS1 and $\mathbf{h S 2}$ ) and hydroxyl-free (S1 and S2) glass surfaces

\begin{tabular}{llllll}
\hline & Hydroxylated & & & \multicolumn{2}{l}{ Hydroxyl-free } \\
\cline { 2 - 3 } \cline { 5 - 6 } & hS1 & hS2 & & S1 & S2 \\
\hline$E_{\text {ad }}$ & $13.5(200)$ & $10.8(160)$ & & $40.2(594)$ & $33.3(492)$ \\
$E_{\text {dis }}$ & $11.9(176)$ & $10.4(154)$ & & $12.7(188)$ & $12.8(190)$
\end{tabular}

However, the reactivity of a single $\mathrm{Au}$ atom and small $\mathrm{Au}_{n}$ clusters for $n<5$ is significantly higher than that of larger clusters or two-dimensional Au layers. ${ }^{53-56}$ In line with our findings for the hydroxylated glass surface, the DFT calculations of Del Vitto et al. ${ }^{20}$ using cluster models of amorphous silica and periodic models of an all-silica edingtonite surface found very weak binding energies of less than $10 \mathrm{~kJ}(\mathrm{~mol} \mathrm{Au})^{-1}$ for an isolated $\mathrm{Au}$ atom and an $\mathrm{Au}$ dimer interacting with bridging Si-O-Si and terminal silanol groups. A similar result has been obtained for the adsorption of an Au atom on defectfree unsupported and supported crystalline silica films. ${ }^{21,23}$ In contrast, for models of hydroxyl-free silica surfaces ${ }^{20,22}$ and defective crystalline silica films ${ }^{23}$ a very strong interaction of a single $\mathrm{Au}$ atom with surface defect sites has been predicted, 305-380 kJ (mol Au) ${ }^{-1}$ for $\mathrm{Si}^{3}$ and $208-300 \mathrm{~kJ}(\mathrm{~mol} \mathrm{Au})^{-1}$ for NBO. The interaction energy decreases significantly with increasing number of $\mathrm{Au}$ atoms. For $\mathrm{Au}_{2}$ the reported calculated binding energies are 93-124 $\mathrm{kJ}(\mathrm{mol} \mathrm{Au})^{-1}$ for $\mathrm{Si}^{3}$ and $70-140 \mathrm{~kJ}(\mathrm{~mol} \mathrm{Au})^{-1}$ for NBO centers. ${ }^{20,22,23}$ Due to already mentioned high reactivity of individual $\mathrm{Au}$ atoms and of $\mathrm{Au}$ clusters these interaction energies are significantly higher than Au adhesion energies derived in the present work ( $c f$. Table 5).

\section{Conclusions}

The results of our simulations demonstrate that properties of silica glass can be accurately described employing relatively small semi-amorphous periodic models. Simulation cells containing only $64 \mathrm{SiO}_{2}$ units properly reproduce experimental structural parameters, mechanical properties and IR vibrational spectra of bulk silica glass. The validity of the glass models is further confirmed by good agreement between the calculated and the experimental number of silanol and germinal silanol groups for hydroxylated silica glass surface models constructed from the semi-amorphous cells. $A b$ initio MD simulations of an Au monolayer deposited on hydroxyl-free and hydroxylated glass surface models point to a substantial 
surface dependence of Au agglomeration (solid-state dewetting) behavior. On hydroxyl-free surfaces, Au monolayers undergo virtually instantaneous agglomeration, which is accompanied by the formation of voids exposing a bare silica glass surface. In contrast, simulated annealing of Au monolayers on hydroxylated surface models reveals a significantly higher structural stability. This points to a key role of reactive defect sites such as $\mathrm{Si}^{3}$ and $\mathrm{NBO}$ centers in the kinetics of solid-state dewetting processes of metals deposited on the glass surface. Such defect sites are important for initial void nucleation and formation of metal clusters. The same Au agglomeration behavior is observed for two different surface models with substantially different concentrations and locations of surface defects. This is a strong indication that our conclusion concerning the important role of defect sites in solid-state dewetting processes on the glass surface remains valid even in the case of lower concentrations of surface defects observed in experiments. In addition, the present calculations demonstrate the crucial role of appropriate inclusion of dispersion interactions in DFT simulations of metals deposited on the glass surface. For defective, hydroxyl-free glass surfaces the dispersion correction accounts for $35 \%$ of the total adhesion energy. This effect is even more dramatic for hydroxylated glass surfaces, where the adhesion energies are almost entirely due to the dispersion interactions. This finding is in line with a recent report by Ruiz Puigdollers et $a l .{ }^{57}$ for metal clusters on oxide surfaces. Finally, the gold adhesion energies of 200 and $160 \mathrm{~kJ}\left(\mathrm{~mol} \mathrm{~nm}^{2}\right)^{-1}$ calculated for hydroxylated glass surfaces are in good agreement with the experimental data.

\section{Acknowledgements}

Authors gratefully acknowledge financial support from the Ministry of Education, Science and Culture of the state Thuringia through the European Regional Development Fund (project NANOSOR), the Fonds der Chemischen Industrie and the Turbomole GmbH.

\section{References}

1 L. Armelao, D. Barreca, G. Bottaro, A. Gasparotto, S. Gross, C. Maragno and E. Tondello, Coord. Chem. Rev., 2006, 250, 1294-1314.

2 C. Burda, X. Chen, R. Narayanan and M. A. El-Sayed, Chem. Rev., 2005, 105, 1025-1102.

3 D. Gentili, G. Foschi, F. Valle, M. Cavallini and F. Biscarini, Chem. Soc. Rev., 2012, 41, 4430-4443.

4 A. L. Giermann and C. V. Thompson, J. Appl. Phys., 2011, 109, 083520.

5 C. V. Thompson, Annu. Rev. Mater. Res., 2012, 42, 399-434.

6 L. Yao and J. He, Prog. Mater. Sci., 2014, 61, 94-143.

7 J. Ye and C. V. Thompson, Adv. Mater., 2011, 23, 1567-1571.

8 D. Wang, R. Ji and P. Schaaf, Beilstein J. Nanotechnol., 2011, 2, 318-326.

9 M. A. Schmidt, D. Y. Lei, L. Wondraczek, V. Nazabal and S. A. Maier, Nat. Commun., 2012, 3, 1108.
10 R. Meszaros, B. Merle, M. Wild, K. Durst, M. Göken and L. Wondraczek, Thin Solid Films, 2012, 520, 7130-7135.

11 R. Meszaros, M. Wild and L. Wondraczek, Glass Technol.: Eur. J. Glass Sci. Technol., Part A, 2013, 54, 177-184.

12 R. E. Hummel, R. T. Dehoff, S. Matts-Goho and W. M. Goho, Thin Solid Films, 1981, 78, 1-14.

13 E. Jiran and C. V. Thompson, J. Electron. Mater., 1990, 19, 1153-1160.

14 E. Jiran and C. V. Thompson, Thin Solid Films, 1992, 208, 23-28.

15 J.-Y. Kwon, T.-S. Yoon, K.-B. Kim and S.-H. Min, J. Appl. Phys., 2003, 93, 3270-3278.

16 C. M. Müller and R. Spolenak, Acta Mater., 2010, 58, 6035-6045.

17 C. M. Müller and R. Spolenak, J. Appl. Phys., 2013, 113, 094301.

18 G. Seguini, J. Llamoja Curi, S. Spiga, G. Tallarida, C. Wiemer and M. Perego, Nanotechnology, 2014, 25, 495603.

19 C.-L. Kuo and P. Clancy, Modell. Simul. Mater. Sci. Eng., 2005, 13, 1309-1329.

20 A. Del Vitto, G. Pacchioni, K. H. Lim, N. Rösch, J.-M. Antonietti, M. Michalski, U. Heiz and H. Jones, J. Phys. Chem. B, 2005, 109, 19876-19884.

21 L. Giordano, A. Del Vitto and G. Pacchioni, J. Chem. Phys., 2006, 124, 034701.

22 K. H. Lim, O. Zakharieva, A. M. Shor and N. Rösch, Chem. Phys. Lett., 2007, 444, 280-286.

23 U. Martinez, L. Giordano and G. Pacchioni, J. Phys. Chem. B, 2006, 110, 17015-17023.

24 A. C. T. van Duin, S. Dasgupta, F. Lorant and W. A. Goddard III, J. Phys. Chem. A, 2001, 105, 9396-9409.

25 A. C. T. van Duin, A. Strachan, S. Stewman, Q. Zhang, X. Xu and W. A. Goddard III, J. Phys. Chem. A, 2003, 107, 3803-3811.

26 S. Plimpton, J. Comp. Physiol., 1995, 117, 1-19.

27 J. P. Perdew, K. Burke and M. Ernzerhof, Phys. Rev. Lett., 1996, 77, 3865-3868.

28 P. E. Blöchl, Phys. Rev. B: Condens. Matter Mater. Phys., 1994, 50, 17953-17979.

29 G. Kresse and D. Joubert, Phys. Rev. B: Condens. Matter Mater. Phys., 1999, 59, 1758-1775.

30 G. Kresse and J. Hafner, Phys. Rev. B: Condens. Matter Mater. Phys., 1993, 47, 558-561.

31 G. Kresse and J. Hafner, Phys. Rev. B: Condens. Matter Mater. Phys., 1994, 49, 14251-14269.

32 G. Kresse and J. Furthmüller, Comput. Mater. Sci., 1996, 6, 15-50.

33 G. Kresse and J. Furthmüller, Phys. Rev. B: Condens. Matter Mater. Phys., 1996, 54, 11169-11186.

34 H. J. Monkhorst and J. D. Pack, Phys. Rev. B: Condens. Matter Mater. Phys., 1976, 13, 5188-5192.

35 S. Grimme, J. Antony, S. Ehrlich and H. Krieg, J. Chem. Phys., 2010, 132, 154104.

$36 \mathrm{X} . \mathrm{Wu}$, D. Vanderbilt and D. R. Hamann, Phys. Rev. B: Condens. Matter Mater. Phys., 2005, 72, 035105.

37 S. Baroni, S. de Gironcoli and A. Dal Corso, Rev. Mod. Phys., 2001, 73, 515-562. 
38 A. P. Scott and L. Radom, J. Phys. Chem., 1996, 100, 16502-16513.

39 C. T. Kirk, Phys. Rev. B: Condens. Matter Mater. Phys., 1988, 38, 1255-1273.

40 A. C. Wright, J. Non-Cryst. Solids, 1994, 179, 84-115.

41 J. R. G. da Silva, D. G. Pinatti, C. E. Anderson and M. L. Rudee, Philos. Mag., 1975, 31, 713-717.

42 H. F. Poulsen, J. Neuefeind, H.-B. Neumann, J. R. Schneider and M. D. Zeidler, J. Non-Cryst. Solids, 1995, 188, 63-74.

43 W. Pabst and E. Gregorová, Ceram.-Silik., 2013, 57, 167-184.

44 L. T. Zhuravlev, Colloids Surf., A, 2000, 173, 1-38.

45 A. Grabbe, T. A. Michalske and W. L. Smith, J. Phys. Chem., 1995, 99, 4648-4654.

46 D. W. Sindorf and G. E. Maciel, J. Phys. Chem., 1982, 86, 5208-5219.

47 C. C. Liu and G. E. Maciel, J. Am. Chem. Soc., 1996, 118, 5103-5119.

48 B. C. Bunker, D. M. Haaland, T. A. Michalske and W. L. Smith, Surf. Sci., 1989, 222, 95-118.
49 F. L. Galeener, Solid State Commun., 1982, 44, 1037-1040.

50 A. A. Bobyshev and V. A. Radzig, Kinet. Catal., 1988, 29, 551-559.

51 V. A. Radzig, in Defects in $\mathrm{SiO}_{2}$ and Related Dielectrics: Science and Technology, ed. G. Pacchioni, L. Skuja and D. L. Griscom, Kluwer Academic, Dordrecht, 2000, pp. 339-370.

52 J. M. McCrate and J. G. Ekerdt, Chem. Mater., 2014, 26, 2166-2171.

53 M. Gao, A. Lyalin, M. Takagi, S. Maeda and T. Taketsugu, J. Phys. Chem. C, 2015, 119, 11120-11130.

54 M. Boronat, A. Leyva-Pérez and A. Corma, Acc. Chem. Res., 2014, 47, 834-844.

55 J. J. Determan, S. Moncho, E. N. Brothers and B. G. Janesko, J. Phys. Chem. C, 2014, 118, 15693-15704.

56 B. Yoon, P. Koskinen, B. Huber, O. Kostko, B. von Issendorff, H. Häkkinen, M. Moseler and U. Landman, ChemPhysChem, 2007, 8, 157-161.

57 A. Ruiz Puigdollers, P. Schlexer and G. Pacchioni, J. Phys. Chem. C, 2015, 119, 15381-15389. 